\title{
(2) OPEN ACCESS \\ Weight cycling is associated with adverse cardiometabolic markers in a cross-sectional representative US sample
}

\author{
Lisa Kakinami $\mathbb{C}_{1}{ }^{1,2}$ Bärbel Knäuper, ${ }^{3}$ Jennifer Brunet ${ }^{4}$
}

\begin{abstract}
${ }^{1}$ Mathematics and Statistics, Concordia University, Montreal, Quebec, Canada ${ }^{2}$ PERFORM Centre, Montreal, Canada

${ }^{3}$ McGill University, Montreal, Quebec, Canada

${ }^{4}$ University of Ottawa, Ottawa, Ontario, Canada
\end{abstract}

Correspondence to Dr Lisa Kakinami, Mathematics and Statistics, Concordia University, Montreal, Quebec H3G 1M8, Canada; lisa.kakinami@concordia.ca

Received 25 October 2019 Revised 27 February 2020 Accepted 14 April 2020
Check for updates

(C) Author(s) (or their employer(s)) 2020. Re-use permitted under CC BY-NC No commercial re-use. See rights and permissions. Published by BMJ.

To cite: Kakinami L,

Knäuper B, Brunet J. J

Epidemiol Community

Health 2020;74:662-667.

\begin{abstract}
Background Whether weight cycling (repeated weight loss and regain) is associated with cardiometabolic health is unclear. Study objective was to examine whether weight cycling since young adulthood (ie, 25 years of age) was associated with cardiometabolic markers.

Methods Data from a nationally representative crosssectional US sample (National Health and Nutrition Examination Survey, 1999-2014) were used. Weight history was based on self-reported weight at age 25, 10 years prior and 1 year prior to the survey $(n=4190,51 \%$ male). Using current self-reported weight as the anchor, participants were classified as (1) stable weight, (2) weight losers, (3) weight gainers and (4) weight cyclers. Cardiometabolic markers included fasting lipids, insulin sensitivity and blood pressure. Multiple linear regressions were used to analyse weight history (reference: stable weight) and adjusted for covariates. Analyses incorporated the sampling design and survey weights and were stratified by sex or weight status.

Results Compared with females with stable weight, female weight cyclers had worse lipids and homeostasis model assessment for insulin resistance (HOMA-IR) (all ps<0.05). Compared with males with stable weight, male weight cyclers had worse high-density lipoprotein cholesterol (HDL) and HOMA-IR (ps<0.05). Weight cyclers with normal weight had worse HDL and low-density lipoprotein cholesterol (ps<0.05), and weight cyclers with overweight or obesity had worse HOMA-IR ( $p=0.05)$. Blood pressure was not associated.

Conclusion Weight cycling is adversely associated with cardiometabolic markers but associations differ by sex and weight status. While weight cycling is consistently associated with worse cardiometabolic markers among females, results are mixed among males. Weight cycling is associated with worse lipid measures for normal weight persons, and marginally worse insulin sensitivity for those with overweight/obesity.
\end{abstract}

\section{INTRODUCTION}

Upon making behavioural changes with the intention of losing weight, an estimated $30 \%$ to $50 \%$ of adults report significant weight loss (ie, $>5 \%$ of their body weight). ${ }^{12}$ However, approximately onethird of adults who lose weight regain some or all of it back, ${ }^{13}$ which can lead to repeated attempts to lose weight with subsequent regain. Indeed, an estimated $20 \%$ to $30 \%$ of adults experience repeated cycles of weight loss and regain-a phenomenon referred to as weight cycling. ${ }^{4}$ Whereas excess weight across the lifespan is a significant global health problem because it is a risk factor for a variety of chronic conditions, ${ }^{5}$ the impact of weight cycling remains unclear. ${ }^{6-9}$ Some studies have shown that weight cycling is associated with type II diabetes and multiple cardiometabolic risk factors, ${ }^{10-12}$ whereas others have not found associations. ${ }^{13}{ }^{14}$ Part of the inconsistency might be in the duration of weight history: studies reporting no significant associations had shorter periods of weight history being captured. A further delineation into mild, moderate or severe weight cycling based on the number of cycling episodes and associating it with cardiometabolic health also yielded inconsistent results. ${ }^{1415}$

Mehta et $a l^{9}$ have argued that methodological differences have reduced the evidentiary support of a relationship between weight cycling and adverse cardiometabolic risk. In some studies, researchers compared weight cyclers to all non-cyclers (thus grouping together participants who either lost, gained or maintained weight); in such instances, no associations were observed. ${ }^{13}{ }^{14}$ In contrast, studies in which these groupings were kept separate reported that weight cycling was associated with greater cardiometabolic risk than weight loss. ${ }^{10} 16$ Additional limitations include small or nonrepresentative cross-sectional samples. ${ }^{9}$

The primary objective of this study was to investigate whether weight cycling since young adulthood (ie, 25 years of age) was associated with cardiometabolic markers at 50 years of age (on average) in a large representative US sample. The secondary objective was to investigate whether there was a differential impact between mild and severe weight cycling.

\section{METHODS}

\section{Study sample}

Data were from the National Health and Nutrition Examination Survey (NHANES), a series of large cross-sectional surveys administered continuously since 1999 in 2-year cycles. Using a stratified, multistage probability sampling design, a representative sample of civilian non-institutionalised adults living in the United States was measured. Approval for NHANES was obtained from the National Center for Health Statistics (NCHS) Research Ethics Review Board (Protocol \#98-12, \#2005-06 and \#201117). All participants provided informed consent and participated in a single survey cycle. Data collected from the 1999-2014 cycles were used $(\mathrm{n}=82$ 091).

As the study objective was to investigate the association between weight cycling and cardiometabolic 
markers from age 25 onward for a minimum of 10 years, participants aged $<35$ years were excluded from the analyses $(n=49$ 847). Other exclusion criteria were consistent with prior studies, ${ }^{9}{ }^{17}$ resulting in a final analytic sample size of 4910 (figure 1).

\section{Measures}

Body mass and height

At the time of the survey, height and body mass (referred to as weight hereon) were measured by study staff following standardised procedures. ${ }^{18}$ Height was measured with a stadiometer (calibrated weekly), and weight was measured with a digital scale (calibrated daily). These measures were used to calculate body mass index (BMI) and to categorise participants as having normal weight $\left(\mathrm{BMI}<25 \mathrm{~kg} / \mathrm{m}^{2}\right)$, overweight $\left(\geq 25\right.$ and $\left.<30 \mathrm{~kg} / \mathrm{m}^{2}\right)$ or obesity $\left(\geq 30 \mathrm{~kg} / \mathrm{m}^{2}\right)$ at the time of the survey.

\section{Weight history}

Participants self-reported their weight at the time of the survey as well as their weight 1 year prior to the survey, 10 years prior to the survey and at age 25 years. Using these self-reported data, participants were categorised as follows: (1) those with stable weight if all previous time points were within 5\% of their (self-reported) weight at the time of the survey; (2) weight losers if their weight at the time of the survey was $\geq 5 \%$ less than previously, and they had no history of gaining weight; (3) weight gainers if their weight at the time of the survey was $\geq 5 \%$ more than previously, and they had no history of losing weight; and (4) weight cyclers if their weight fluctuated between higher and lower than their weight at the time of the survey but did not meet previous definitions of stable weight, weight losers or weight gainers. A secondary analysis further subdivided the weight cyclers into (1) mild if weight at the time of the survey differed by $\geq 5 \%$ from one of the previous time points or (2) severe if weight differed by $\geq 5 \%$ at two or more of the previous time

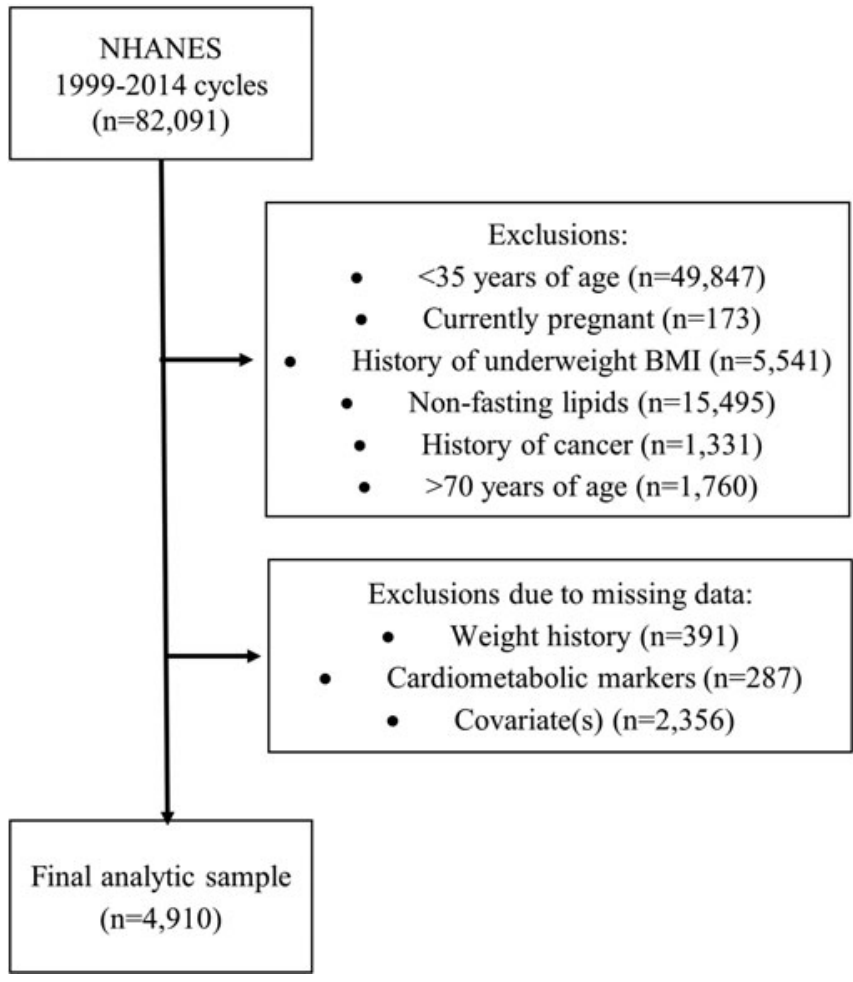

Figure 1 Study flow diagram. points. As there is no consensus on operationalising weight cycling or severe weight cycling, these categorisations were analytic decisions made by the research team. The implications of this are described in the 'Discussion' section.

\section{Cardiometabolic markers}

A phlebotomist collected blood samples via venipuncture using standardised NCHS procedures. Participants were instructed to fast for 10-12 hours. Samples were analysed for total cholesterol (TC), high-density lipoprotein cholesterol (HDL) and triglycerides (TG) (measured in $\mathrm{mg} / \mathrm{dL}$ ). Insulin resistance was assessed by homeostasis model assessment (HOMA-IR). ${ }^{19} 20$ Samples were processed immediately or placed on ice and stored at $-20^{\circ} \mathrm{C}$ (if shipped for analysis within 1 month) or $-80^{\circ} \mathrm{C}$ (if shipped for analysis more than 1 month later). Low-density lipoprotein cholesterol (LDL) was estimated using the Friedewald equation. ${ }^{21}$ Due to laboratory changes over time, all cardiometabolic values were calibrated based on the recommended regressions to be comparable to one another. $^{22}$ Notably, scientists have cautioned against drawing conclusions from HDL differences between 1999 and $2008^{22}$; thus, a sensitivity analysis excluding data collected during these years was conducted. Systolic (SBP) and diastolic blood pressure (DBP) were measured on the right arm with a sphygmomanometer after 5 min in a resting, seated position. Cuff size was determined based on arm circumference and measured in triplicate (or quadruplicate). After excluding the first measure, the mean SBP and DBP were calculated.

\section{Covariates}

Demographic characteristics, current medication use and past year's household income (in US dollars) were all selfreported. Household income was compared to poverty guidelines by the Department of Health and Human Services to calculate poverty-to-income ratios (PIRs), ${ }^{23}$ whereby larger values indicate higher incomes. The ratios adjust for household size, geographic region and inflation. Lifestyle behaviours such as current smoking status (yes/no) and minutes of physical activity in the past week were also self-reported. Minutes of moderate-tovigorous intensity physical activity (MVPA) measured using the International Physical Activity Questionnaire were truncated to $180 \mathrm{~min} /$ day as recommended. ${ }^{24}$ Other covariates included the discrepancy between self-reported weight and measured weight at the time of the survey, and the largest fluctuation in weight (difference between highest and lowest weight).

\section{Statistical analysis}

All analyses were conducted with SAS 9.4 and accounted for the complex sampling design. Sampling weights were used to account for non-response and were either fasting weights (for the LDL, TG, HOMA, SBP and DBP outcomes) or full sample weights (for TC and HDL) as recommended in the NHANES analytic guidelines. Statistical tests (ie, $\chi^{2}$ and t-tests) were conducted to compare the analytic sample and excluded participants, and for describing differences between weight history groupings (ie, stable weight, weight losers, weight gainers, weight cyclers).

Using stable weight as the reference group, the associations between weight cycling history and cardiometabolic markers were analysed using separate multiple linear regression models. Models adjusted for age, sex, race, current smoking status, household PIR, minutes of MVPA in the past week, largest weight fluctuation ([highest weight-lowest weight/lowest weight] $\times$ 100) and the difference between self-reported weight and measured weight at the time of the survey. Whether participants were 
currently on medications for high lipids or inadequate glucose control was additionally included as a covariate in the models with TC, HDL, LDL, TG and HOMA-IR as the dependent variables, respectively. Models for SBP and DBP additionally adjusted for whether the person was currently taking medications to treat high blood pressure. In each model, contrast statements tested for significant differences between weight cyclers and weight gainers.

Tests for interaction between weight history with sex or current weight status were conducted. As significant interactions were detected, all analyses were stratified by sex and weight status (based on measured BMI at the time of the survey). These analyses adjusted for all covariates listed above (except for sex and weight status, as appropriate). No significant interactions between weight cycling history and age were detected. Results from the full 1999-2014 data collection cycles are uniformly presented as sensitivity analyses using only the HDL data from 2008 to 2014 were largely unchanged from the main findings.

\section{RESULTS}

The final analytic sample differed from the excluded sample in sex $(51 \%$ male vs 49\%; $\mathrm{P}<0.05)$, age (50.9 years vs 29.3 , respectively; $\mathrm{P}<0.05)$ and BMI $\left(29.9\right.$ vs $25.0 \mathrm{~kg} / \mathrm{m}^{2}$, respectively; $\mathrm{P}<0.05)$. The weighted mean age for the analytic sample was 50.9 , which suggests the spacing between measurements between age 25 and 10 years prior approximates 15 years. In the analytic sample, approximately $8 \%$ had stable weight, $5 \%$ were weight losers, $53 \%$ were weight gainers and 33\% were weight cyclers.
Weight history was significantly associated with demographic characteristics and cardiometabolic markers (table 1).

\section{Weight cycling and cardiometabolic markers}

In multiple linear regression analyses, female weight cyclers and weight gainers had worse cardiometabolic markers compared with those with stable weight (table 2). For instance, compared with females with stable weight, female weight cyclers had worse lipid profiles (betas: $11.66,-7.58,14.95$ and $19.66 \mathrm{mg} / \mathrm{dL}$ for TC, HDL, LDL and TG, respectively; all ps $<0.05$ ), as well as in their HOMA-IR (beta: 0.85, respectively, $\mathrm{p}<0.05$ ). Similarly, compared with females with stable weight, female weight gainers had worse lipid profiles (betas: 11.30, -8.70, 14.52 and $26.26 \mathrm{mg} / \mathrm{dL}$ for TC, HDL, LDL and TG, respectively; all ps $<0.05$ ), as well as in their HOMA-IR (beta: 1.55 , respectively, $\mathrm{p}<0.05$ ). From the subsequent contrast statements, the magnitude of the difference in HOMA-IR between weight cyclers and weight gainers (ie, beta: 0.85 in HOMA-IR for weight cyclers, and beta: 1.55 in HOMA-IR for weight gainers) was significant. No other contrast statements between female weight cyclers and female weight gainers were significant. The risks from weight cycling among men were associated with HDL (beta: 4.97, respectively, $\mathrm{p}<0.05$ ) and HOMA-IR (beta: 1.15, respectively, $\mathrm{p}<0.0001)$.

When stratifying the models by current weight status (ie, status at the time of the survey), compared with those with stable weight, risks from weight cycling largely persisted for lipid

\begin{tabular}{|c|c|c|c|c|c|}
\hline & Stable* $(n=381)$ & Losers* $(n=271)$ & Gainers* $(n=2558)$ & Cyclers*,$+(n=1700)$ & $P$ value \\
\hline Male, \% & $70.6 \%$ & $59.3 \%$ & $48.9 \%$ & $47.9 \%$ & $<0.0001$ \\
\hline Age in years, $M(S D)$ & $49.3(0.6)$ & $49.8(0.8)$ & $50.7(0.2)$ & $51.9(0.3)$ & 0.001 \\
\hline \multicolumn{6}{|l|}{ Race, \% } \\
\hline African-American & $5 \%$ & $6 \%$ & $52 \%$ & $37 \%$ & 0.0008 \\
\hline Caucasian & $9 \%$ & $5 \%$ & $53 \%$ & $33 \%$ & \\
\hline Hispanic & $6 \%$ & $4 \%$ & $54 \%$ & $36 \%$ & \\
\hline Other & $12 \%$ & $5 \%$ & $54 \%$ & $29 \%$ & \\
\hline Current measured BMI in $\mathrm{kg} / \mathrm{m}^{2}, \mathrm{M}(\mathrm{SD})$ & $24.6(0.2)$ & $26.0(0.3)$ & $30.6(0.2)$ & $30.1(0.2)$ & $<0.0001$ \\
\hline PIR, M (SD) & $3.5(0.09)$ & $2.9(0.14)$ & $3.1(0.06)$ & $3.1(0.06)$ & $<0.0001$ \\
\hline MVPA minutes per week, M (SD) & $136.4(12.2)$ & $115.6(18.4)$ & $95.7(4.8)$ & $115.7(6.1)$ & $<0.0001$ \\
\hline Difference between measured and self-reported weight $\dagger$ & $-0.3(0.4)$ & $-0.9(0.4)$ & $-1.9(0.2)$ & $-1.7(0.2)$ & 0.005 \\
\hline Highest weight in pounds, M (SD) & $184.7(2.2)$ & $220.5(12.3)$ & $208.5(7.6)$ & $248.3(21.0)$ & 0.02 \\
\hline Lowest weight in pounds, M (SD) & $161.0(2.0)$ & $166.3(2.3)$ & $149.7(0.7)$ & $154.9(1.1)$ & $<0.0001$ \\
\hline Largest weight fluctuation§, \% & $14.6 \%$ & $32.9 \%$ & $41.6 \%$ & $62.5 \%$ & 0.001 \\
\hline \multicolumn{6}{|l|}{ Cardiometabolic markers, M (SD) } \\
\hline TC & $197.5(2.4)$ & $188.3(2.4)$ & $202.2(1.0)$ & $200.1(1.4)$ & 0.0001 \\
\hline HDL & $57.4(1.0)$ & $58.9(1.1)$ & $52.2(0.4)$ & $53.3(0.6)$ & $<0.0001$ \\
\hline LDL & $116.8(2.1)$ & $110.0(2.5)$ & $122.7(0.9)$ & $121.1(1.1)$ & 0.0001 \\
\hline TG & $115.9(4.8)$ & $98.1(2.8)$ & $135.1(1.9)$ & $128.3(2.8)$ & $<0.0001$ \\
\hline HOMA-IR & $2.0(0.1)$ & $2.0(0.1)$ & $3.7(0.1)$ & $3.4(0.1)$ & $<0.0001$ \\
\hline SBP & $118.3(0.9)$ & $120.6(1.3)$ & $122.4(0.4)$ & $122.3(0.5)$ & 0.0003 \\
\hline DBP & $71.1(0.6)$ & $69.4(1.1)$ & $72.9(0.3)$ & $71.3(0.5)$ & 0.0005 \\
\hline
\end{tabular}

Boldface indicates statistical significance $(p<0.05)$

* Mean, SE unless otherwise indicated; Percentages may not add up to 100 due to rounding.

tAmong the 1700 weight cyclers, 1592 were mild weight cyclers (weight at the time of the survey differed by at least $5 \%$ from one previous weight measure and the person did not consistently gain or lose weight at all time points) and 108 were severe weight cyclers (weight at the time of the survey differed by at least $5 \%$ from at least two previous weight measures, and the person did not consistently gain or lose weight at all time points).

¥Defined as measured weight- self-reported weight at the time of the study.

$\S$ Defined as ((highest weight-lowest weight)/lowest weight) $\times 100$.

BMI, body mass index; DBP, diastolic blood pressure; HDL, high-density lipoprotein cholesterol; HOMA, homeostasis model assessment; LDL, low-density lipoprotein cholesterol; MVPA, moderate-to-vigorous physical activity; PIR, poverty-to-income ratio; SBP, systolic blood pressure; TC, total cholesterol; TG, triglycerides. 
Table 2 Multivariable linear regressions on cardiometabolic measures, National Health and Nutrition Examination Survey, 1999-2014 ( $\mathrm{n}=4910)$

\begin{tabular}{|c|c|c|c|c|c|c|c|}
\hline & $\mathrm{TC}+\ldots \mathrm{B}(\mathrm{SE})$ & HDLt, $\neq$ B (SE) & LDLt, $¥ B$ (SE) & $\mathrm{TGt}, \neq \mathrm{B}(\mathrm{SE})$ & HOMAt,§ B (SE) & SBPt, П B (SE) & DBPt, П B (SE) \\
\hline \multicolumn{8}{|c|}{ Males only $(n=2490)^{* *}$} \\
\hline Stable & Reference & Reference & Reference & Reference & Reference & Reference & Reference \\
\hline Losers & $-9.69(5.2)$ & $2.01(1.7)$ & $-6.56(4.9)$ & $-25.61(8.1)^{* *}$ & $-0.41(0.3)$ & $-1.00(2.2)$ & $-2.50(1.8)$ \\
\hline Gainers & $1.90(3.4)$ & $-6.03(1.1)^{* * *}$ & $4.43(3.1)$ & $17.86(6.9)^{*}$ & $1.60(0.2)^{* * *}$ & $2.42(1.1)^{*}$ & $2.81(1.0)^{* *}$ \\
\hline Cyclers & $-1.50(3.5)$ & $-4.97(1.2)^{* * *}$ & $1.84(3.1)$ & $10.69(7.4)$ & $1.15(0.3)^{* * *}$ & $2.26(1.2)$ & $1.30(1.3)$ \\
\hline \multicolumn{8}{|c|}{ Females only $(n=2420) t \dagger$} \\
\hline Stable & Reference & Reference & Reference & Reference & Reference & Reference & Reference \\
\hline Losers & $-4.53(5.6)$ & $-0.23(2.8)$ & $-2.03(5.3)$ & $-8.43(8.1)$ & $-0.11(0.2)$ & $4.13(2.6)$ & $-0.54(1.4)$ \\
\hline Gainers & $11.30(4.3)^{*}$ & $-8.70(1.8)^{* * *}$ & $14.52(4.0)^{* *}$ & $26.26(6.2)^{* * *}$ & $1.55(0.1)^{* * *}$ & $3.77(1.6)^{*}$ & $1.28(1.0)$ \\
\hline Cyclers & $11.66(4.5)^{*}$ & $-7.58(1.9)^{* *}$ & $14.95(4.0)^{* *}$ & $19.66(6.1)^{* *}$ & $0.85(0.2)^{* * *}$ & $3.31(1.7)$ & $0.13(0.9)$ \\
\hline \multicolumn{8}{|c|}{ Normal weight only $(n=1127) \ddagger \ddagger$} \\
\hline Stable & Reference & Reference & Reference & Reference & Reference & Reference & Reference \\
\hline Losers & $-6.02(5.2)$ & $3.02(2.0)$ & $-5.89(4.5)$ & $-13.68(6.1)^{*}$ & $0.08(0.1)$ & $0.94(2.5)$ & $-0.63(1.4)$ \\
\hline Gainers & $4.29(4.0)$ & $-4.38(1.5)^{* *}$ & $6.86(3.5)^{*}$ & $9.29(5.5)$ & $0.27(0.09)^{* *}$ & $1.72(1.3)$ & $1.30(1.1)$ \\
\hline Cyclers & $7.51(4.1)$ & $-3.67(1.7)^{*}$ & $10.72(3.3)^{* *}$ & $1.37(6.1)$ & $0.12(0.08)$ & $-0.82(1.4)$ & $0.42(1.1)$ \\
\hline \multicolumn{8}{|c|}{ Overweight/obese only $(n=3783) \S \S$} \\
\hline Stable & Reference & Reference & Reference & Reference & Reference & Reference & Reference \\
\hline Losers & $-13.10(5.7)^{*}$ & $1.38(2.1)$ & $-7.60(5.9)$ & $-33.32(11.6)^{* *}$ & $-0.63(0.3)$ & $0.10(2.0)$ & $-2.94(1.7)$ \\
\hline Gainers & $-1.03(4.0)$ & $-2.66(1.3)^{*}$ & $0.67(3.9)$ & $3.50(10.7)$ & $1.26(0.3)^{* *}$ & $1.63(1.2)$ & $1.57(1.0)$ \\
\hline Cyclers & $-3.74(4.1)$ & $-1.93(1.4)$ & $-1.49(4.1)$ & $-1.78(10.6)$ & $0.64(0.3)^{\wedge}$ & $1.40(1.4)$ & $0.19(1.2)$ \\
\hline
\end{tabular}

$\wedge p=0.05{ }^{*} p<0.05 ;{ }^{* *} p<0.01 ;{ }^{* * *} p<0.0001$.

Boldface indicates statistical significance $(p<0.05)$ in contrast statements between weight gain and weight cycling.

tAdjusted for age, sex, race (African-American, Caucasian, Hispanic or Other), whether the person was currently a smoker (yes/no), the household's income-to-poverty ratio, minutes of moderate-to-vigorous physical activity intensity in the past week, percentage difference between the highest and lowest weight, and the difference between self-reported weight and measured weight at the time of the survey.

¥Additionally adjusted for whether the person was currently on lipid medication

§Additionally adjusted for whether the person was currently on glucose or insulin medication.

TAdditionally adjusted for whether the person was currently on medication for hypertension.

**Weight losers $(n=163)$, stable weight $(n=254)$, weight gainers $(n=1249)$, weight cyclers $(n=824)$.

t+Weight losers $(n=108)$, stable weight $(n=127)$, weight gainers $(n=1309)$, weight cyclers $(n=876)$.

$\neq \neq$ Weight losers $(n=137)$, stable weight $(n=242)$, weight gainers $(n=420)$, weight cyclers $(n=328)$.

$\S \S$ Weight losers $(n=134)$, stable weight $(n=139)$, weight gainers $(n=2138)$, weight cyclers $(n=1372)$

DBP, diastolic blood pressure; HDL, high-density lipoprotein cholesterol; HOMA, homeostasis model assessment; LDL, low-density lipoprotein cholesterol; SBP, systolic blood pressure; TC, total cholesterol; TG, triglycerides.

measures for normal weight persons, and persisted in insulin sensitivity measures for those with overweight/obesity. Weight cycling was not associated with blood pressure in any of the models.

\section{Severity of weight cycling and cardiometabolic risk}

Among the 1700 weight cyclers, nearly all (94\%) were mild cyclers, and $6 \%$ were severe weight cyclers. Considering the very small sample size, analyses based on severity of weight cycling could not be conducted.

\section{DISCUSSION}

In this large, representative sample of the US population, adults with stable weight had better cardiometabolic profiles than did adults who weight cycled. However, the impact of weight cycling on insulin markers appears to be smaller than the impact of weight gain. Collectively, these results underscore the importance of assessing different types of weight histories to properly investigate their associated cardiometabolic impact.

The magnitude of the impact of weight cycling on cardiometabolic markers observed in this study was approximately 5\% to $10 \%$ higher compared with maintaining a stable weight. As a point of comparison, pharmacological interventions (eg, statins) are associated with lipid differences of $30 \%$ to $50 \%{ }^{25}$ However, results are aligned with the literature on lipids' differences based on non-pharmacological interventions (eg, diet and exercise) and represent clinically meaningful risks. ${ }^{26}$ One potential pathway that is hypothesised is that weight cycling promotes visceral fat accumulation, ${ }^{27}$ which thereby leads to greater cardiometabolic risks.

Notably, the current study demonstrated that among participants who were considered overweight or with obesity, weight cycling was not associated with most lipid measures, but was marginally associated with insulin sensitivity. In fact, the risks from weight gain were larger than those from weight cycling in persons with overweight or obesity. This is consistent with the literature among both human populations, as well as animal models. ${ }^{28}{ }^{29}$ Indeed, an important emerging finding in the literature is that weight cycling may not be as adverse for health than sustained obesity. ${ }^{28} 30$ For instance, cardiometabolic changes among people who regained weight 4 -years post-intervention from the Look AHEAD weight-loss trial were not deemed to be worse than changes among those who did not initially lose weight. ${ }^{8}$

In contrast, among participants who were considered normal weight, in this study, weight cycling was associated with poorer lipid measures. As there are multiple pathways through which diabetes and cardiovascular disease are linked (eg, inflammation, oxidative stress and dyslipidemia), results suggest a different biological pathway between lipid measures and diabetes risk. ${ }^{31}$ Further research on whether adverse metabolic measures 
progress to dyslipidemia among weight cyclers with overweight or obesity is needed. Results from this study suggest that while weight cycling is adversely associated with cardiometabolic measures, key differences based on sex and weight status exist. Future studies further exploring these associations in large, heterogeneous diverse samples are needed to better elucidate these relationships.

The study's second objective was to investigate mild and severe weight cycling on cardiometabolic risk. Due to the small number of severe weight cyclers, this objective could not be addressed. This may have been due to the operationalisation of severe weight cycling herein; however, consensus in the literature on defining weight cycling severity is lacking. Severity of weight cycling has been operationalised as losing $\geq 20 \mathrm{lb}$ on three or more occasions, as well as losing $\geq 11 \mathrm{lb}$ on at least three or more occasions with weight regain. ${ }^{15} 32$ Similarly, weight cycling definitions differ in the literature. Associations between weight cycling and cardiometabolic risk have been shown to differ based on whether weight cycling is measured in absolute or relative terms. ${ }^{13}$ This study operationalised weight cycling based on relative weight changes using percentage fluctuations, rather than absolute weight changes. Using relative weight changes better accounts for people of various heights and weights from this diverse population and is aligned with measurable outcomes in weight loss intervention studies. ${ }^{8}$ In addition, advancements in statistical modelling have enabled researchers to define weight history based on complex, non-linear trajectories over time. ${ }^{30}$ While these non-linear trajectories are likely superior in model fit, differences in operationalisations depend on the data on hand and results across studies cannot be easily compared. Accordingly, it is important to come to a consensus on how to operationalise weight cycling that can be easily translated and interpreted to allow for comparisons across studies.

This study has limitations that should be acknowledged. Whether weight loss was intentional was not directly measured; intentional weight loss likely results in larger changes in diet and physical activity, which may both independently be associated with cardiometabolic measures. ${ }^{33}$ While we did adjust for physical activity, eating patterns were not measured and thus could not be incorporated into the analysis. Weight history was selfreported, which renders it subject to bias and could have led to misclassification when categorising participants into weight cycling groups. Thus, while this study aimed to incorporate a long history of weight, it could only incorporate longer periods of defining weight history based on retrospective recalls. However, although imperfect, having self-reported weight and height at different ages allowed us to look at interconstruct change, whereas using objectively measured weight and height could exacerbate purported validity problems considering that differences in measures may invoke differential categories. The definition and operationalisation of weight cycling as well as weight cycling severity were limited in scope and cannot fully account for variation of weight across the entire timeframe of interest. Additional research on weight history including better definitions, prevalent estimates and motivations for weight cycling is needed. In particular, a recent publication reported that maximum BMI was a better predictor of mortality than current BMI. ${ }^{34}$ Indeed, in our study, weight gainers had the highest maximum weight (weight cyclers had the second highest maximum weight), while weight cyclers had the largest fluctuation between highest and lowest weight. Whether the higher cardiometabolic risk factors from weight cycling are due to this previous history of maximum weight or an underlying mechanism due to weight fluctuations should be further explored.

As temporality cannot be inferred due to the study design, whether weight cycling is worse for health than sustained obesity could not be investigated. Such an investigation using data from a cohort with a large representation of people with weight cycling and/or sustained obesity weight histories would make an important contribution to the literature. Relatedly, although current weight could theoretically be incorporated into the statistical analysis, it was not adjusted for in the current study. This was an analytic decision made due to the likelihood that current weight may also lie in the causal pathway between weight history and cardiometabolic health. ${ }^{35}$ Differences were observed between the analytical and excluded samples. In particular, adults who were $<35$ years of age were excluded, limiting the ability of the study results to be generalised to younger adults. Lastly, the results suggest that both sex and weight status may be modifiers in the relationship between weight history and cardiometabolic risk factors. However, this study was not able to assess for these relationships while stratifying by sex and weight status simultaneously due to insufficient sample sizes. Further research on these relationships is needed.

Despite the aforementioned limitations, the current study has several notable strengths. These include the large, representative sample of adults living in the United States. Data were collected from NHANES, a well-established research programme with a methodologically rigorous protocol, data collection and data cleaning procedures. This study is among the few studies to incorporate weight history spanning a minimum of 10 years and to analyse whether the associations differed by sex and weight status after adjusting for a number of important covariates.

In summary, the results indicate that weight cycling is adversely associated with several cardiometabolic markers. Furthermore, the magnitude of the impact is similar to the impact of weight gain (without weight loss) and depends on sex and weight status. In order to determine whether the impact is significantly greater based on the duration and severity of weight cycling, more longitudinal research with varying weight statuses and histories is needed.

\section{What is already known on this subject}

- Approximately one-third of adults who lose weight will regain some or all of it back. Repeated attempts to lose weight (with subsequent weight regain) is known as weight cycling. Weight cycling has been inconsistently associated with cardiometabolic risk factors.

\section{What this study adds}

- Compared with those with stable weight, cyclers had worse cardiometabolic profiles. Associations differed based on sex and weight status.

Acknowledgements LK holds a Junior 1 salary award from Fonds de Recherche du Québec - Santé. JB and BK have no financial disclosures to declare. The authors thank Stefanie Marchione for her assistance with the preliminary data analysis. Portions of these data were presented at the sixth Canadian Obesity Summit (Ottawa, April 23-26, 2019). 
Contributors LK performed the statistical analysis, interpreted the data and wrote the first draft. BK and JB contributed to the interpretation of the results, reviewed the manuscript and provided substantive feedback. All authors were involved with the conception of the study and approved the final manuscript.

Funding LK holds a Junior 1 salary award from Fonds de Recherche du Québec Santé. The funders were not involved in the study design, data analyses, data interpretation, manuscript writing or submission processes.

Competing interests None declared.

Patient consent for publication Not required.

Data sharing statement Data are available in a public, open-access repository.

Provenance and peer review Not commissioned; externally peer reviewed.

Open access This is an open access article distributed in accordance with the Creative Commons Attribution Non Commercial (CC BY-NC 4.0) license, which permits others to distribute, remix, adapt, build upon this work non-commercially, and license their derivative works on different terms, provided the original work is properly cited, appropriate credit is given, any changes made indicated, and the use is noncommercial. See: http://creativecommons.org/licenses/by-nc/4.0/.

\section{ORCID iD}

Lisa Kakinami http://orcid.org/0000-0002-8496-7643

\section{REFERENCES}

1 McGuire MT, Wing RR, Hill JO. The prevalence of weight loss maintenance among American adults. Int J Obes Relat Metab Disord 1999:23:1314-19.

2 Phelan S, Wing RR, Loria CM, et al. Prevalence and predictors of weight-loss maintenance in a biracial cohort: results from the coronary artery risk development in young adults study. Am J Prev Med 2010;39:546-54.

3 Weiss EC, Galuska DA, Kettel KL, et al. Weight regain in U.S. adults who experienced substantial weight loss, 1999-2002. Am J Prev Med 2007;33:34-40.

4 Mackie GM, Samocha-Bonet D, Tam CS. Does weight cycling promote obesity and metabolic risk factors? Obes Res Clin Pract 2017;11:131-9.

5 Kivimäki M, Kuosma E, Ferrie JE, et al. Overweight, obesity, and risk of cardiometabolic multimorbidity: pooled analysis of individual-level data for 120813 adults from 16 cohort studies from the USA and Europe. Lancet Public Health 2017.

6 Kodama S, Fujihara K, Ishiguro H, et al. Unstable bodyweight and incident type 2 diabetes mellitus: a meta-analysis. J Diabetes Investig 2017;8:501-9.

7 Truesdale KP, Stevens J, Cai J. The effect of weight history on glucose and lipids. Am J Epidemiol 2005;161:1133-43.

8 Wing R, Espeland M, Clark J, et al. Association of weight loss maintenance and weight regain on 4-year changes in CVD risk factors: the action for health in diabetes (Look AHEAD) clinical trial. Diabetes Care 2016;39:1345-55.

9 Mehta T, Smith DL, Muhammad J, et al. Impact of weight cycling on risk of morbidity and mortality. Obes Rev Off J Int Assoc Study Obes 2014;15:870-81.

10 Lee D-H, Steffes MW, Gross M, et al. Differential associations of weight dynamics with coronary artery calcium versus common carotid artery intima-media thickness: the CARDIA study. Am J Epidemiol 2010;172:180-9.

11 Kataja-Tuomola M, Sundell J, Männistö S, et al. Short-term weight change and fluctuation as risk factors for type 2 diabetes in Finnish male smokers. Eur J Epidemiol 2010;25:333-9.

12 Vergnaud A-C, Bertrais S, Oppert J-M, et al. Weight fluctuations and risk for metabolic syndrome in an adult cohort. Int J Obes 2005: 2008. 32: 315-21.

13 Graci S, Izzo G, Savino S, et al. Weight cycling and cardiovascular risk factors in obesity. Int J Obes Relat Metab Disord 2004;28:65-71.
14 Field AE, Manson JE, Laird N, et al. Weight cycling and the risk of developing type 2 diabetes among adult women in the United States. Obes Res 2004:12:267-74.

15 Mason C, Foster-Schubert KE, Imayama I, et al. History of weight cycling does not impede future weight loss or metabolic improvements in postmenopausal women. Metabolism 2013;62:127-36

16 Blüher M, Rudich A, Klöting N, et al. Two patterns of adipokine and other biomarker dynamics in a long-term weight loss intervention. Diabetes Care 2012;35:342-9.

17 Waring ME, Eaton CB, Lasater TM, et al. Incident diabetes in relation to weight patterns during middle age. Am J Epidemiol 2010;171:550-6.

18 Centers for Disease Control and Prevention (CDC). National Center for Health Statistics (NCHS). National Health and Nutrition Examination Survey data. Hyattsville, MD: U.S. Department of Health and Human Services, Centers for Disease Control and Prevention, 1999.

19 Matthews DR, Hosker JP, Rudenski AS, et al. Homeostasis model assessment: insulin resistance and beta-cell function from fasting plasma glucose and insulin concentrations in man. Diabetologia 1985;28:412-19.

20 Katz A, Nambi SS, Mather K, et al. Quantitative insulin sensitivity check index: a simple, accurate method for assessing insulin sensitivity in humans. I Clin Endocrinol Metab 2000;85:2402-10.

21 Friedewald WT, Levy RI, Fredrickson DS. Estimation of the concentration of low-density lipoprotein cholesterol in plasma, without use of the preparative ultracentrifuge. Clin Chem 1972:18:499-502.

22 National Health and Nutrition Examination Survey: 1999-2000 data documentation, codebook, and frequencies. 2010. Available https://wwwn.cdc.gov/Nchs/Nhanes/ 1999-2000/LAB13.htm (accessed 28 Jan 2019).

23 U.S. Department of health and human services. poverty guidelines, research, and measurement. Washington, DC: U.S. Department of Health and Human Services, 2013.

24 Guidelines for data processing and analysis of the International Physical Activity Questionnaire (IPAQ). 2005. Available https://docs.google.com/viewer?a=v\&pid=sites\& srcid=ZGVmYXVsdGRvbWFpbnx0aGVpcGFxfGd40jEONDgxMDk3NDU1YWRIZTM (accessed 24 Feb 2020).

25 Waters DD, Boekholdt SM. An evidence-based guide to cholesterol-lowering guidelines. Can J Cardiol 2017;33:343-9.

26 Bruckert E, Rosenbaum D. Lowering LDL-cholesterol through diet: potential role in the statin era. Curr Opin Lipidol 2011;22:43.

27 Zeigler ZS, Birchfield N, Moreno K, et al. Fatness and fluctuating body weight: effect on central vasculature. Biores Open Access 2018;7:90-100.

28 Smith DL, Yang Y, Nagy TR, et al. Weight cycling increases longevity compared with sustained obesity in Mice. Obesity 2018;26:1733-9.

29 Wing RR, Jeffery RW, Hellerstedt WL. A prospective study of effects of weight cycling on cardiovascular risk factors. Arch Intern Med 1995;155:1416-22.

30 Cologne J, Takahashi I, French B, et al. Association of weight fluctuation with mortality in Japanese adults. JAMA Netw Open 2019;2:e190731.

31 Dokken BB. The pathophysiology of cardiovascular disease and diabetes: beyond blood pressure and lipids. Diabetes Spectr 2008;21:160-5.

32 Lahti-Koski M, Männistö S, Pietinen P, et al. Prevalence of weight cycling and its relation to health indicators in Finland. Obes Res 2005;13:333-41.

33 O'Connor LE, Li J, Sayer RD, et al. Short-term effects of healthy eating pattern cycling on cardiovascular disease risk factors: pooled results from two randomized controlled trials. Nutrients 2018;10:1725

34 Stokes A, Preston SH. Revealing the burden of obesity using weight histories. Proc Nat Acad Sci U S A 2016;113:572-7.

35 Chiolero A, Kaufman IS, Paradis G. Why adjustment for current weight can bias the estimate of the effect of birth weight on blood pressure: shedding light using causal graphs. J Hypertens 2012;30:1042-5. 